\section{It May Be Time to Abandon Urine Tests for Bladder Cancer}

\author{
Joseph J. Fantony, MD, and Brant A. Inman, MD, MS
}

Bladder cancer is the second most common malignancy of the genitourinary tract and fifth most common malignancy overall in the United States. ${ }^{1}$ The current standard for diagnosis and surveillance of bladder cancer includes a combination of cystoscopy and urine cytology. ${ }^{2}$ The propensity of bladder cancer for recurrence leads to a need for frequent monitoring, making bladder cancer currently the most expensive per-patient malignancy to treat in the United States. ${ }^{3}$

These points, along with the discomfort, anxiety, and morbidity of frequent cystoscopy have led to much effort in developing alternative, less-invasive methods for bladder surveillance. ${ }^{4}$ In this commentary, we suggest that no advancement in noninvasive testing has occurred in recent years capable of altering the current endoscopic surveillance scheme. We further argue that the poor performance, marginal clinical utility, and potential harm of the currently available urine tests make them inadequate for regular clinical use.

\section{Poor "Real-World" Performance}

Two of the most common urine-based tests involved in the diagnosis and surveillance of bladder cancer are urine cytology and the fluorescence in situ hybridization (FISH) assay. Urine cytology involves microscopic examination and morphologic description of exfoliated urothelial cells by a qualified pathologist, and the FISH assay detects several chromosomal abnormalities that are known to be associated with the development of urothelial carcinoma (UC). The literature varies widely on the performance metrics of these tests, depending on the tested cohort and how the tests are implemented.

Urine cytology is commonly thought to be highly specific (86\%-96\%) but with poor sensitivity $(16 \%-86 \%)$, particularly in detecting low-grade tumors. ${ }^{5,6}$ With most tumors being low-grade at diagnosis, cytology is poor as a screening test. ${ }^{7,8}$ However, because of its reported high specificity, it continues to be used to help in the detection of high-grade lesions, including carcinoma in situ.

We recently conducted a large retrospective analysis of more than 5000 urine cytology tests performed at Duke University Medical Center over a 10-year period to determine its testing performance in our patient population. We found that the performance of urine cytology depended greatly on how the atypical and suspicious results were viewed. When the results were considered to be "positive," the sensitivity was approximately $65 \%$ and the specificity approximately $45 \%$. In contrast, when the results were considered "negative," the sensitivity was approximately $15 \%$ and the specificity approximately 95\%. Given that over the last 10 years approximately half of our urine cytology results landed in the suspicious/atypical "grey-zone" of equivocation, these results are particularly concerning. When a test is often equivocal and its performance depends dramatically on how equivocal results are viewed, this results in a situation wherein patient harm can occur.

With these issues in mind, we also assessed the performance of FISH testing in our patient population. In a similar analysis of more than $3500 \mathrm{FISH}$ tests, we found a $37 \%$ sensitivity and $84 \%$ specificity in detecting bladder cancer. This can be compared with the oft-quoted sensitivity of approximately $70 \%$ and specificity around $90 \%$ to $100 \%$, depending on the grade of the tumor. ${ }^{9,10}$ Proponents of the FISH test claim
Joseph J. Fantony, MD

Joseph J. Fantony, MD, is a senior resident in Urology at Duke University Medical Center. He received his undergraduate education at Norwich University, and accepted a commission in the United States Army. He earned his medical degree from University of Massachusetts, and following this he completed his general surgery internship at Walter Reed National Military Medical Center. Most recently, he completed a 1 year research fellowship under the guidance of Dr. Brant A. Inman at Duke University. Upon graduation from the Duke Urology residency program, he plans on entering a urologic oncology fellowship.

The ideas and viewpoints expressed in this commentary are those of the author and do not necessarily represent any policy, position, or program of NCCN. 


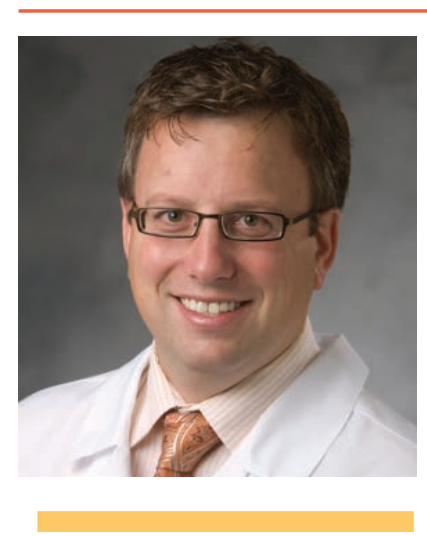

Brant A. Inman, MD, MS

Brant A. Inman, MD, MS, is an Associate Professor of Urology at the Duke Cancer Institute of Duke University Medical Center. Dr. Inman received his undergraduate and medical degrees from the University of Alberta. He then completed his urology residency at Laval University and his urologic oncology fellowship at the Mayo Clinic. Dr. Inman joined Duke in 2008 and is currently Vice Chief of Urology and Director of Clinical Trials for the Division of Urology. His area of clinical expertise is the surgical treatment of genitourinary cancers, with a particular emphasis on bladder cancer.

Dr. Inman conducts research focused on developing novel therapies and diagnostic tests for bladder cancer, with a particular interest in immunotherapy and hyperthermia. Dr. Inman's clinical trial portfolio includes trials of novel diagnostic tests and immunotherapies for bladder and prostate cancer.

Nationally, Dr. Inman is a member of the NCCN Guidelines panels for bladder and penile cancers and is also part of the Board of Directors of the Society of Urological Oncology (SUO) Clinical Trials Committee and the Bladder Cancer subcommittee. He is Vice Chair of the Content Review Committee for the American Urological Association (AUA) and Finance Committee member for the Southeastern Section of the American Urological Association (SESAUA). Dr. Inman also currently serves as consulting editor for European Urology. that its specificity improves if you follow up with patients for longer periods, because it detects cancer earlier than cystoscopy. In other words, some claim that false-positive FISH results are often true "anticipatory positive" results. To determine the presence of anticipatory positive testing properties, we allowed our cystoscopy result to lag up to 390 days. Therefore, a test result that was initially false-positive could become a true-positive test if a subsequent cystoscopy became positive in the ensuing 390 days. We saw no improvement in the sensitivity or specificity over the course of the year, suggesting that a positive FISH result associated with a negative cystoscopy result is usually a false-positive result.

Another consideration with both cytology and FISH is spectrum bias. That is, do the tests perform differently in different patient subgroups? We found that advancing age, male gender, and smoking history all resulted in a change in diagnostic test performance. This means that urine tests for bladder cancer have different sensitivities and specificities depending on the patient, which is an undesirable feature for a diagnostic test for cancer.

These analyses reveal an inherent problem with urinary diagnostic testing, which is the difficulty of translating test performance to real-world populations being screened for bladder cancer. For this reason, it is important to assess the ability of a given test to perform as marketed in each physician's patient cohort and, at our large medical center, the ability of these tests to detect bladder cancer in our patients is clearly inadequate.

\section{Replacement for Cystoscopy}

For a urine test to replace cystoscopy as the gold standard for detecting bladder cancer, it must be superior to cystoscopy in at least 1 of 3 ways: cost, diagnostic accuracy, and/or invasiveness. With respect to cost, cytology and FISH add significant expense to the diagnosis and surveillance of bladder cancer, while rarely adding unique information that would not be garnered from cystoscopy and upper tract imaging alone. ${ }^{11-13}$ As mentioned previously, the diagnostic accuracy is not adequate for either test, and neither seems to have any significant anticipatory positive properties that would alter a typical surveillance regime. We agree that the invasive nature of cystoscopy causes discomfort to the patient in the clinic, but in our experience what causes the patient the most discomfort and anxiety is a positive urine test occurring in the context of a normal cystoscopy and normal upper tract imaging. Patients dwell on these results and feel that a cancer is slowly eating away at them, undiagnosed. Worse, these equivocal or discordant results can lead to unnecessary and potentially harmful procedures.

\section{Potential Harm}

The potential for avoidable iatrogenic complications is introduced in the situation of a positive or equivocal (atypical or suspicious) urine test result in the context of a negative cystoscopy result. This is because at many institutions the urine test will lead to pressure to investigate further, and this normally means bladder biopsies, urethral biopsies, axial imaging, retrograde pyelography, or ureteroscopy. Moreover, if selective ureteral urine samples are obtained and either of these has an abnormal result, a second surgical procedure with ureteroscopy will usually be considered. These procedures require preoperative counseling, medical optimization, and usually general or spinal anesthesia, all of which take time, cost money, and ultimately put the patient at risk for iatrogenic injury.

This is of particular importance given that the decision for further investigation is 
often based on the result of a relatively inaccurate urine test. Most urologists remember a patient or 2 in whom they have caused an iatrogenic bladder or ureteral injury from an endoscopic "low-risk" procedure. Even more common are postprocedure urinary tract infections, sometimes associated with treatment-resistant bacteria and occasionally leading to sepsis. Many (possibly most) of these complications occur in patients who do not have cancer and who were evaluated for a false-positive urine test result. These are risks caused by overtreatment that are avoidable and preventable.

\section{Utility}

These issues raise the question of whether these urine tests have clinical utility. Does the result change what you will ultimately do for the patient? And if it does, should it? The answer to this question really depends on the provider's institution and individual practices. Patients undergoing evaluation for asymptomatic microscopic hematuria have an approximately $1 \%$ to $2 \%$ rate of being diagnosed with a urothelial malignancy. ${ }^{14}$ Because the pretest probability of cancer is so low in these patients, most positive urine tests will be false-positive. Fortunately, the American Urological Association now recommends against the routine use of urine cancer tests in this population. ${ }^{15}$ Patients with painless gross hematuria have about a $15 \%$ to $20 \%$ rate of urothelial malignancy, and a positive or equivocal cytology or positive FISH result with an otherwise normal workup (CT urography and office cystoscopy) creates a difficult decision point of determining the need for further investigation. ${ }^{16}$

Another group of patients in whom these tests are used frequently are those undergoing surveillance for previously treated bladder cancer. The recurrence rate of bladder cancer varies greatly depending on several risks factors (eg, stage, grade, size, number of tumors); however, in general, recurrence is common (30\%-60\%). Current surveillance schemes require regular cystoscopic evaluation of the bladder (up to every 3 months in patients at high risk) and imaging of the upper tracts (at diagnosis and periodically depending on risk). Because cystoscopy has sensitivity and specificity for detecting bladder cancer of more than $95 \%$ and CT urography has sensitivity and specificity of approximately $95 \%$ for diagnosing upper urinary tract cancer, the probability of missing an upper tract urothelial malignancy is very small. ${ }^{16,17}$ What do urine tests add then, especially since, in our experience, they are far less accurate in the general population than in study cohorts? Probably not that much, except risk for overtreatment and iatrogenic harm.

\section{Conclusions}

At Duke University Medical Center, urinary tests for bladder cancer perform far worse than has been reported in the literature. They do not appear to provide any anticipatory positive properties to alert providers to an early diagnosis of urothelial carcinoma, and their improved performance in subgroups at higher risk is not compelling because these patients undergo screening with better tests anyway. A positive or equivocal urine test is usually not helpful and often leads to unnecessary diagnostic and surgical procedures with inherent cost and risk. Therefore, we contend that until a urine test can be developed that dramatically changes the follow-up regime or replaces office cystoscopy, urine tests have minimal utility and should not be routinely used. 


\section{References}

1. Howlader N, Krapcho M, Garshell J, et al, eds. SEER Cancer Statistics Factsheets: Bladder Cancer. National Cancer Institute. Bethesda, MD, 2015. Available at http://seer.cancer.gov/statfacts/html/urinb.html. Accessed August 11, 2015.

2. Clark PE, Agarwal N, Biagioli MC, et al. Bladder cancer. J Natl Compr Canc Netw 2013;11:446-475.

3. Svatek RS, Hollenbeck BK, Holmang S, et al. The economics of bladder cancer: costs and considerations of caring for this disease. Eur Urol 2014;66:253-262.

4. van der Aa MN, Steyerberg EW, Sen EF, et al. Patients' perceived burden of cystoscopic and urinary surveillance of bladder cancer: a randomized comparison. BJU Int 2008;101:1106-1110.

5. Lokeshwar VB, Habuchi T, Grossman HB, et al. Bladder tumor markers beyond cytology: International Consensus Panel on bladder tumor markers. Urology 2005;66(6 Suppl 1):35-63.

6. Yafi FA, Brimo F, Steinberg J, et al. Prospective analysis of sensitivity and specificity of urinary cytology and other urinary biomarkers for bladder cancer. Urol Oncol 2015;33:66:e25-31.

7. Sylvester RJ, van der Meijden A, Witjes JA, et al. High-grade Ta urothelial carcinoma and carcinoma in situ of the bladder. Urology 2005;66(6 Suppl 1):90-107.

8. Fleshner NE, Herr HW, Stewart AK, et al. The National Cancer Data Base report on bladder carcinoma. The American College of Surgeons Commission on Cancer and the American Cancer Society. Cancer 1996;78:15051513.

9. Dimashkieh H, Wolff DJ, Smith TM, et al. Evaluation of urovysion and cytology for bladder cancer detection: a study of 1835 paired urine samples with clinical and histologic correlation. Cancer Cytopathol 2013;121:591-597.

10. Bubendorf L, Grilli B, Sauter G, et al. Multiprobe FISH for enhanced detection of bladder cancer in voided urine specimens and bladder washings. Am J Clin Pathol 2001;116:79-86.

11. Chen GJ, Amiel GE, Roville K, et al. Cost implication of using Urovysion in surveillance care for noninvasive bladder cancer [abstract]. J Urol 2009;181:Abstract 197.

12. Fritsche HM, Burger M, Dietmaier W, et al. Multicolor FISH (UroVysion) facilitates follow-up of patients with high-grade urothelial carcinoma of the bladder. Am J Clin Pathol 2010;134: 597-603.

13. Trivedi D, Messing EM. Commentary: the role of cytologic analysis of voided urine in the work-up of asymptomatic microhematuria. BMC Urol 2009;9:13.

14. Loo RK, Lieberman SF, Slezak JM, et al. Stratifying risk of urinary tract malignant tumors in patients with asymptomatic microscopic hematuria. Mayo Clin Proc 2013;88:129-138.

15. Davis R, Jones JS, Barocas DA, et al. Diagnosis, evaluation and follow-up of asymptomatic microhematuria $(\mathrm{AMH})$ in adults: AUA guideline. J Urol 2012;188(6 Suppl):2473-2481.

16. Blick CG, Nazir SA, Mallett S, et al. Evaluation of diagnostic strategies for bladder cancer using computed tomography (CT) urography, flexible cystoscopy and voided urine cytology: results for 778 patients from a hospital haematuria clinic. BJU Int 2012;110:84-94.

17. Cowan NC, Turney BW, Taylor NJ, et al. Multidetector computed tomography urography for diagnosing upper urinary tract urothelial tumour. BJU Int 2007;99:1363-1370. 